\title{
Erratum: Entanglement Detection beyond Measuring Fidelities [Phys. Rev. Lett. 124, 200502 (2020)]
}

\author{
M. Weilenmann๑, B. Dive๑, D. Trillo®, E. A. Aguilar®, and M. Navascués®
}

(Q) (Received 13 August 2020; published 7 October 2020)

DOI: 10.1103/PhysRevLett.125.159903

It was brought to our attention that Table I of our Letter contains several errors in the fraction of states that are positive partial transpose (PPT) as well as in the fraction that is certified to be unfaithful with our criterion. In the following, we provide the corrected table.

TABLE I. Percentage of unfaithful states detected via SDP 2 and the PPT condition when sampling uniformly according to either the Hilbert-Schmidt metric $(H S)$ or the Bures metric $(B)$ (sampling $10^{6}$ points for each dimension in each case). $d$ denotes the local dimension of each subsystem of the bipartite system. Note that we are actually interested in $\mathcal{U}_{2} \backslash \mathcal{S} \supseteq \tilde{\mathcal{U}}_{2} \backslash \mathcal{S}^{1}$, which may be even larger.

\begin{tabular}{lcccc}
\hline \hline$d$ & $\mathcal{S}^{1}(H S)$ & $\tilde{\mathcal{U}}_{2} \backslash \mathcal{S}^{1}(H S)$ & $\mathcal{S}^{1}(B)$ & $\tilde{\mathcal{U}}_{2} \backslash \mathcal{S}^{1}(B)$ \\
\hline 2 & $24.2 \%$ & $21.2 \%$ & $7.4 \%$ & $15.4 \%$ \\
3 & $0.01 \%$ & $94.5 \%$ & $0 \%$ & $54.8 \%$ \\
4 & $0 \%$ & $100 \%$ & $0 \%$ & $97.0 \%$ \\
5 & $0 \%$ & $100 \%$ & $0 \%$ & $100 \%$ \\
\hline \hline
\end{tabular}

The biggest change is that the proportion of unfaithful states sampled according to the Bures metric is considerably smaller for $d=3$, than what the previous version of the table indicated. However, these changes do not affect the conclusions of our Letter. We verified that the original code used to generate this data (provided at [1]) works as intended; it therefore remains unchanged.

We thank Otfried Gühne for pointing out the errors in Table I of our article. This work was supported by the Austrian Science fund (FWF) stand-alone Project No. P 30947.

[1] M. Weilenmann, B. Dive, D. Trillo, E. A. Aguilar, and M. Navascués, Github repository for entanglement detection beyond measuring fidelities, https://github.com/BenDive/Entanglement-Detection-Beyond-Measuring-Fidelities. 